\title{
TLR4 induces tumor growth and inhibits paclitaxel activity in MyD88-positive human ovarian carcinoma in vitro
}

\author{
AN-CONG WANG ${ }^{1,2}$, YUE-BING MA ${ }^{2}$, FENG-XIA WU ${ }^{3}$, ZHI-FANG MA $^{2}$, NAI-FU LIU $^{2}$, \\ RONG GAO ${ }^{2}$, YONG-SHENG GAO ${ }^{4}$ and XIU-GUI SHENG ${ }^{2}$ \\ ${ }^{1}$ Department of Obstetrics and Gynecology, Linyi People's Hospital, Linyi, Shandong 276002; \\ ${ }^{2}$ Department of Gynecologic Oncology, Shandong Cancer Hospital and Institute, Jinan, \\ Shandong 250117; ${ }^{3}$ Department of Anatomy, Shandong University, Jinan, Shandong 250012; \\ ${ }^{4}$ Department of Pathology, Shandong Cancer Hospital and Institute, Jinan, Shandong 250117, P.R. China
}

Received May 22, 2013; Accepted December 3, 2013

DOI: $10.3892 / 01.2013 .1759$

\begin{abstract}
In ovarian cancer patients, chemotherapy resistance is the principal factor restricting long-term treatment. Paclitaxel (Pac) has been previously reported to be a ligand to Toll-like receptor 4 (TLR4). It was determined that TLR4 signaling is divided into the following two pathways: Myeloid differentiation factor 88 (MyD88)-dependent and MyD88-independent. The present study investigated the effect of TLR4 ligation by Pac in MyD88-positive (MyD88+) and MyD88-negative (MyD88') human ovarian cancer cell lines. An RNA interference expression vector was specifically constructed to target TLR4 mRNA, which was stably transfected into the human ovarian cancer cell lines (SKOV3, OVCAR3, A2780 and 3AO). Cytokines, including interleukin (IL)-6 and IL-8, were detected. Cell proliferation and apoptosis were assessed in the cells transfected with scramble control and TLR4 shRNA to explore the possible functions of TLR4 in ovarian cancer cell growth. It was found that lipopolysaccharide and Pac significantly increase the secretion of IL-6 and IL-8 in the SKOV3 cell line. Similarly, Pac resulted in a significant upregulation of IL-6 and IL-8 in OVCAR3 cells, but not in A2780 and 3AO cells. These results suggested that in $\mathrm{MyD}^{+} 8^{+}$ovarian cancer cell lines, TLR4 depletion shows increased sensitivity to Pac treatment in inhibiting cell proliferation compared with in cells without TLR4 knockdown. On the contrary, such changes were not found in MyD88 cells (A2780 and 3AO). TLR4 negatively regulates Pac chemotherapy, particularly in terms of cell
\end{abstract}

Correspondence to: Professor Xiu-Gui Sheng, Department of Gynecologic Oncology, Shandong Cancer Hospital and Institute, 440 Jiyan Road, Jinan, Shandong 250117, P.R. China

E-mail: shengxgjnsd@163.com

Abbreviations: TLR4, Toll-like receptor 4; Pac, paclitaxel; MyD88, myeloid differentiation factor 88

Key words: ovarian cancer, TLR4, MyD88, paclitaxel, chemotherapy proliferation, and TLR4 may be a novel treatment target in Pac-resistant ovarian cancer.

\section{Introduction}

Toll-like receptors (TLRs) are a family of pattern recognition receptors. To date, 11 human TLRs and 13 mouse TLRs have been identified (1). Mammalian TLRs recognize microbial products and initiate innate immune responses (2).

Although a limited number of studies have analyzed the correlation between TLR expression and human malignancy, several studies concerning the expression of TLRs and cancer have been conducted (3).

The Toll-related proteins were first identified in mammals (4) and the mammalian TLR4 was rapidly demonstrated to be responsible for the recognition of lipopolysaccharide (LPS) (5). It has been previously reported that TLR4 negatively regulates the Salmonella-induced antitumor activity (6). Notably, TLR4 has been reported to be important in promoting the immune escape of human lung cancer cells by inducing immunosuppressive cytokines and apoptosis resistance (7).

In ovarian cancer patients, chemotherapy resistance is the principal factor restricting long-term treatment (8). Previously, paclitaxel (Pac) has been reported to be a ligand to TLR4 (9). The current study investigated the effects of TLR4 in ovarian cancer, particularly in Pac chemotherapy. Myeloid differentiation factor 88 (MyD88) was originally isolated as a gene that is induced rapidly during the interleukin (IL)-6-stimulated differentiation of M1 myeloleukemic cells into macrophages (10). IL-6 is considered to be involved in host immune responses to types of ovarian cancer $(11,12)$. IL- 6 has also been demonstrated to provide paracrine growth stimulation when monocytes are attracted to types of ovarian cancer that produce macrophage colony-stimulating factor (13). IL-6 signaling in ovarian cancer cells regulates tumor cell proliferation, invasion and angiogenesis $(14,15)$ and IL-8 has also been reported to promote ovarian tumor growth in vivo (16). Previously, it has been reported that TLR4 signaling is divided into the following two pathways: MyD88-dependent and MyD88-independent (17,18). A 
correlation between MyD88 expression and patients' progression-free survival has shown that patients whose tumors do not express MyD88 exhibit a significantly improved progression-free interval compared with patients whose tumors express high levels of MyD88 (19).

The present study investigated the role of TLR4 in ovarian cancer cells and the effect of TLR4 ligand by Pac in MyD88 and MyD88- human ovarian carcinoma in vitro.

\section{Materials and methods}

Reagents. Pac was purchased from Sigma-Aldrich (St. Louis, MO, USA) and the rabbit polyclonal antibodies against TLR4 and MyD88 were purchased from Santa Cruz Biotechnology, Inc., (Santa Cruz, CA, USA).

Cell culture and samples. The in vitro experiments were performed with human ovarian cancer cell lines, SKOV3, OVCAR3, A2780 and 3AO. All the cell lines were obtained from the Basic Research Center, Shandong Cancer Hospital and Institute (Jinan, China). Cells were cultured in RPMI-1640 medium (Gibco-BRL, Carlsbad, CA, USA) supplemented with $10 \%$ heat-inactivated fetal bovine serum (FBS; Gibco-BRL) and incubated under standardized conditions $\left(37^{\circ} \mathrm{C} ; 5 \%\right.$ $\mathrm{CO}_{2}$ atmosphere).

Samples of normal ovarian tissue adjacent to tumor $(n=12)$ and borderline $(n=8)$ and malignant $(n=24)$ tumors were collected with the approval of the Ethics Committee of the Shandong Cancer Hospital and Institute.

RNA isolation and reverse transcription-polymerase chain reaction $(R T-P C R)$. Total RNA was extracted using the TRIzol reagent kit (Invitrogen Life Technologies, Carlsbad, CA, USA) according to the manufacturer's instructions. Reverse transcription was performed using SYBR ExScript RT-PCR kit (Takara Bio, Inc., Shiga, Japan). The following set of primers were used for amplification: i) human TLR4 sense, 5'-AATGGATCAAGGACCAGAGG-3' and antisense, 5'-CAGCCAGCAAGAAGCATCAG-3'; and ii) human MyD88 sense, 5'-CGCCGGATGGTGGTGGTTGT-3' and antisense, 5'-TGTAGTCGCAGACAGTGATGAACC-3'. The primers were used to amplify an 197-bp fragment of TLR4 cDNA and an 186-bp fragment of MyD88 cDNA. The following primers were used for $\beta$-actin: forward, 5'-TTGTATCGTGGAAGGACTCA-3' and reverse, 5'-TGTCATCATATTTGGCAGGTTT-3'. TLR4 was used to amplify a 197-bp fragment. Forty cycles of PCR were performed at $95^{\circ} \mathrm{C}$ for $30 \mathrm{sec}, 63^{\circ} \mathrm{C}$ for $30 \mathrm{sec}$ and $72^{\circ} \mathrm{C}$ for 45 sec. The primers for human MyD88 were used to amplify a 186-bp fragment of MyD88 cDNA. Thirty cycles of PCR were performed at $95^{\circ} \mathrm{C}$ for $30 \mathrm{sec}, 61^{\circ} \mathrm{C}$ for $30 \mathrm{sec}$ and $72^{\circ} \mathrm{C}$ for $45 \mathrm{sec}$.

SDS-PAGE and western blot analysis. Protein was denatured in sample buffer [2.5\% SDS, $10 \%$ glycerol, $5 \% \beta$-mercaptoethanol, $0.15 \mathrm{~mol} / \mathrm{l}$ Tris ( $\mathrm{pH}$ 6.8) and $0.01 \%$ bromophenol blue] and subjected to $10-12 \%$ SDS-PAGE as previously described (6). The following antibodies were used: Rabbit anti-TLR4 (1:1,000), -MyD88 (1:1,000) and -actin (1:10,000) (Santa Cruz Biotechnology, Inc.). Signals were detected using ECL western blotting detection reagents (Pierce Biotechnology, Inc., Rockford, IL, USA) according to the manufacturer's instructions.

Immunohistochemistry. Paraffin sections of tumor tissues were deparaffinized and microwaved while immersed in $0.01 \mathrm{M}$ citrate buffer ( $\mathrm{pH}$ 6.0) for $20 \mathrm{~min}$. Sections were washed with PBS and incubated overnight at $4^{\circ} \mathrm{C}$ with polyclonal rabbit anti-human TLR4 antibody (1:50) or with polyclonal rabbit anti-human MyD88 (1:50; Santa Cruz Biotechnology, Inc.). Following washing, tissues were incubated with horseradish peroxidase-labeled anti-rabbit antibody for $1 \mathrm{~h}$, followed by 3,3'-diaminobenzidine (Dako, Carpinteria, CA, USA). The results for TLR4 and MyD88 expression in tissues were scored by two independent investigators based on the following levels of staining intensity: None (-), weak $(+)$, moderate $(++)$, or strong $(+++)$.

Incubation of tumor cells with TLR4 ligands. In all experiments testing the effects of LPS or Pac on tumor cells, LPS was used at a concentration of $10 \mu \mathrm{g} / \mathrm{ml}$ and Pac at $2 \mu \mathrm{M}$.

Cytokine and chemokine production. Cytokine and chemokine production by the human ovarian cancer cells was determined using a Luminex-100 System (Luminex, Austin, TX, USA). Supernatants of tumor cells seeded in 12-well plates at $5 \times 10^{5}$ cells/well in $1 \mathrm{ml}$ of LPS or Pac medium were collected following $36 \mathrm{~h}$ of incubation. The levels of IL- 6 and IL- 8 were measured using panels of capture antibody-coated beads and the labeled detection antibodies, which were pretested and qualified by the manufacturer to ensure the absence of cross-reactivity. The assay sensitivity varied between 5 and $15 \mathrm{pg} / \mathrm{ml}$.

Caspase-Glo $3 / 7$ assay. In total, $10 \mu \mathrm{g}$ of protein in a $50 \mu 1$ total volume was mixed with $50 \mu 1$ equilibrated Caspase-Glo 3/7 reagents (Promega Corporation, Madison, WI, USA). Following incubation at room temperature for $1 \mathrm{~h}$, luminescence was measured using TD 20/20 luminometer (Turner Designs, Inc., Sunnyvale, CA, USA). Blank values were subtracted and fold increase in activity was calculated based on the activity measured from untreated cells. Each sample was measured in triplicate.

Construction of RNA interference targeting the TLR4 gene in the vector. Small interfering RNA (siRNA) oligonucleotides specifically targeting TLR4 oligonucleotides were confirmed to be valid by the authors and were designed with the following sequences: Sense, 5'-GGTAAGGAATGAGCTAGTA-3' and antisense, 5'-TACTAGCTCATTCCTTACC-3'. The pGenesil-1 negative control vector (Wuhan Genesil Biotechnology Co., Ltd., Wuhan, China) was used as the negative control plasmid in all experiments as previously described (20).

Transcription and production of stable clones. The day prior to transfection, cells were trypsinized and plated at a density of $5 \times 10^{5}$ cells/well in six-well tissue culture plates (Corning Inc., Corning, NY, USA). Cells were rinsed twice with serum-free RPMI-1640 when the density reached $>90 \%$ confluence. Next, pGenesil-shTLR4 (RNA interference expression vectors) or pGenesil-shControl (control vector) and Lipofectamine 2000 mixtures, prepared in OptiMEM 
(Invitrogen Life Technologies), were added. Following $5 \mathrm{~h}$ of incubation, the plasmid-Lipofectamine 2000 mixture was removed and RMPI-1640 plus 10\% FBS and $800 \mu \mathrm{g} / \mathrm{ml}$ geneticin (Invitrogen Life Technologies) for SKOV3 and OVCAR3 cells, $500 \mu \mathrm{g} / \mathrm{ml} \mathrm{G} 418$ for $3 \mathrm{AO}$ cells and $600 \mu \mathrm{g} / \mathrm{ml}$ geneticin for A2780 cells were added. All the non-transfected cells died within 7 days and a number of surviving transfected cells were harvested 21 days later.

MTT assay. Stable clone cells were seeded into 96-well culture plates $(5,000 /$ well $)$ and treated with $2 \mu \mathrm{mol} / 1 \mathrm{Pac}(2 \mu \mathrm{M})$ for $24 \mathrm{~h}$. As the control for normal cell proliferation, $0.1 \%$ ethanol was used. At the end of each treatment, cells were incubated as previously described (21).

Statistical analysis. Data are presented as the mean \pm standard deviation for continuous variables, and the frequency and percentage for categorical variables. Results were statistically evaluated by analysis of variance. SPSS 17.0 software for Windows was used for statistical treatment (SPSS, Inc. Chicago, IL, USA). P<0.05 was considered to indicate a statistically significant difference.

\section{Results}

Expression of TLR4 and MyD88 in ovarian cancer tissues. Expression of TLR4 and MyD88 were assessed in situ on paraffin sections of normal ovarian tissue adjacent to tumor $(n=12)$ and borderline $(n=8)$ and malignant $(n=24)$ tumors. TLR4 was found to exhibit moderate $(++)$ or strong $(+++)$ expression in malignant (20/24) and borderline (5/8) tumors and normal ovarian epithelium (6/12). However, the expression of MyD88 in malignant tumors was considerably greater $(18 / 24)$ than that in normal ovarian tissue (1/8) or in borderline tumors (1/12) (Table I).

Expression of TLR4 and MyD88 signaling adapter protein in epithelial ovarian cancer (EOC) cell lines. Furthermore, the expression of TLR4 and MyD88 in EOC cells was evaluated by RT-PCR. As shown in Fig. 1, the mRNA of TLR4 were expressed in EOC cell lines. However, the expression of MyD88 was found to differ; SKOV3 and OVCAR3 cells were MyD88-positive, while A2780 and 3AO cells were MyD88-negative.

LPS- and Pac-induced cytokine production in MyD88+ cells. Supernatants of the four cell lines exposed to LPS or Pac for $36 \mathrm{~h}$ were analyzed for levels of inflammatory cytokines and growth factors. SKOV3 and OVCAR3 cells constitutively secreted a wide range of cytokines and chemokines, including IL-6 and IL-8. By contrast, A2780 and 3AO cells produced low levels of these cytokines. LPS and Pac significantly increased the secretion of IL-6 and IL- 8 in SKOV3 cells. Similarly, Pac resulted in a significant upregulation of IL-6 and IL- 8 in OVCAR3 cells, but not in A2780 and 3AO cells (Figs. 2 and 3).

Effect of shRNA on TLR4 gene expression. To investigate the potential role of TLR4 in EOC cell lines, pGenesil-shTLR4 directed at nucleotides 2,202-2,220 of TLR4 was used to selectively reduce TLR4 gene expression in EOC cell lines, SKOV3, OVCAR3, A2780 and 3AO. As shown in Fig. 4, the shTLR4 significantly reduced the expression of the TLR4 mRNA and
Table I. Results for TLR4 and MyD88 expression in tissues.

\begin{tabular}{|c|c|c|}
\hline Definite histology & TLR4 & MyD88 \\
\hline \multicolumn{3}{|c|}{ Malignant tumor cases } \\
\hline 1 & ++ & - \\
\hline 2 & +++ & ++ \\
\hline 3 & +++ & ++ \\
\hline 4 & ++ & - \\
\hline 5 & +++ & +++ \\
\hline 6 & ++ & +++ \\
\hline 7 & +++ & ++ \\
\hline 8 & + & +++ \\
\hline 9 & +++ & ++ \\
\hline 10 & ++ & - \\
\hline 11 & ++ & ++ \\
\hline 12 & +++ & ++ \\
\hline 13 & +++ & +++ \\
\hline 14 & +++ & ++ \\
\hline 15 & ++ & + \\
\hline 16 & ++ & ++ \\
\hline 17 & ++ & + \\
\hline 18 & + & ++ \\
\hline 19 & ++ & ++ \\
\hline 20 & ++ & +++ \\
\hline 21 & + & + \\
\hline 22 & ++ & ++ \\
\hline 23 & + & ++ \\
\hline 24 & ++ & +++ \\
\hline \multicolumn{3}{|l|}{ Borderline cases } \\
\hline 1 & ++ & - \\
\hline 2 & ++ & + \\
\hline 3 & + & - \\
\hline 4 & +++ & ++ \\
\hline 5 & ++ & + \\
\hline 6 & + & - \\
\hline 7 & ++ & + \\
\hline 8 & + & + \\
\hline \multicolumn{3}{|c|}{ Normal ovarian epithelium cases } \\
\hline 1 & ++ & + \\
\hline 2 & +++ & ++ \\
\hline 3 & ++ & + \\
\hline 4 & ++ & + \\
\hline 5 & + & + \\
\hline 6 & + & - \\
\hline 7 & ++ & + \\
\hline 8 & + & + \\
\hline 9 & + & - \\
\hline 10 & + & + \\
\hline 11 & ++ & + \\
\hline 12 & + & + \\
\hline P-value & $0.022^{\mathrm{a}}$ & $0.001^{\mathrm{b}}$ \\
\hline
\end{tabular}

Summary of the pathological characteristics of tissues. TLR4 and MyD88 expression were scored by the following levels of staining intensity: none (-); weak $(+)$; moderate $(++)$; or strong $(+++)$. ${ }^{\mathrm{a}} \mathrm{P}=0.022$, vs. the proportion of TLR4-positive expression in normal ovarian epithelium and ${ }^{\mathrm{b}} \mathrm{P}=0.001$, vs. MyD88-positive expression in borderline and normal ovarian epithelium. TLR4, Toll-like receptor 4; MyD88, myeloid differentiation factor 88 . 


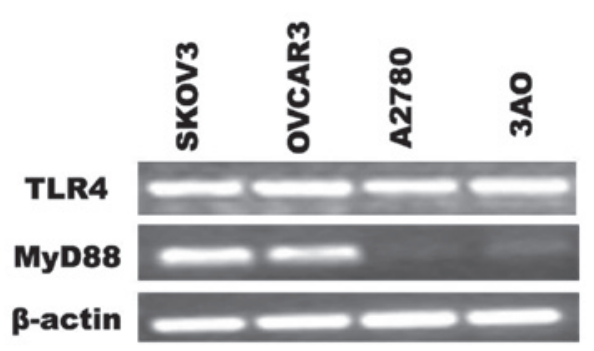

Figure 1. Expression of TLR4 and MyD88 in EOC cells at the mRNA level. TLR4 was expressed in all EOC cell lines; however, positive expression of MyD88 was identified in SKOV3 and OVCAR3 cells, while negative MyD88 expression was observed in A2780 and 3AO cells. TLR4, Toll-like receptor 4; MyD88, myeloid differentiation factor 88; EOC, epithelial ovarian cancer.

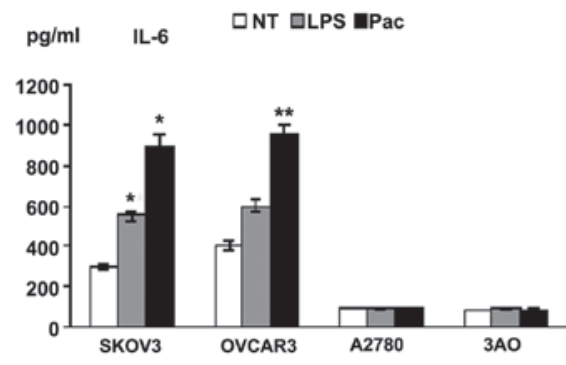

Figure 2. Levels of cytokines in tumor cell SNs. SNs were collected from tumor cells cultured at a density of $5 \times 10^{5}$ cells/well following $36 \mathrm{~h}$ of treatment and tested for levels of IL-6. Three independent experiments were performed. ${ }^{*} \mathrm{P}<0.05$, vs. NT/SKOV3 and ${ }^{* *} \mathrm{P}<0.05$, vs. NT/OVCAR3. SNs, supernatants; IL, interleukin; LPS, lipopolysaccharide; Pac, paclitaxel. NT, cells not treated with LPS or Pac.

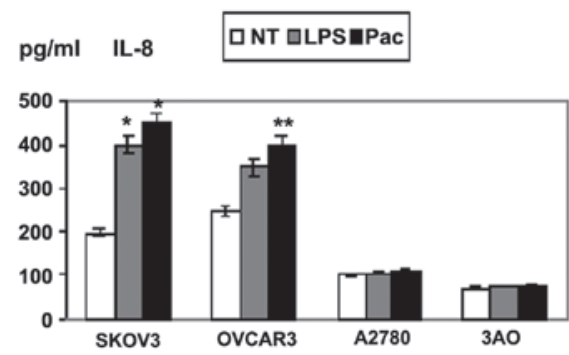

Figure 3. Levels of cytokines in tumor cell SNs. SNs were collected from tumor cells cultured at a density of $5 \times 10^{5}$ cells/well following $36 \mathrm{~h}$ of treatment and tested for levels of IL-8. Three independent experiments were performed. ${ }^{*} \mathrm{P}<0.05$, vs. NT/SKOV3 and ${ }^{* *} \mathrm{P}<0.05$, vs. NT/OVCAR3. SNs, supernatants; IL, interleukin; LPS, lipopolysaccharide; Pac, paclitaxel. NT, cells not treated with LPS or Pac.

protein in these cells (SKOV3/shTLR4, OVCAR3/shTLR4, A2780/shTLR4 and 3AO/shTLR4). The control-scrambled sequence, shControl, exhibited no effect on TLR4 gene expression in cells (SKOV3/shControl, OVCAR3/shControl, A2780/shControl and 3AO/shControl). Thus, the application of TLR4-directed shTLR4 was an effective and selective method of long-term suppression of endogenous TLR4 levels, making it possible to determine in experiments the role of endogeneous TLR4 on EOC cells.

Knockdown of TLR4 depressed cytokine production in $M y D 88^{+}$cells. Supernatants of the four cell lines exposed to LPS or Pac for $36 \mathrm{~h}$ were analyzed for levels of inflam-

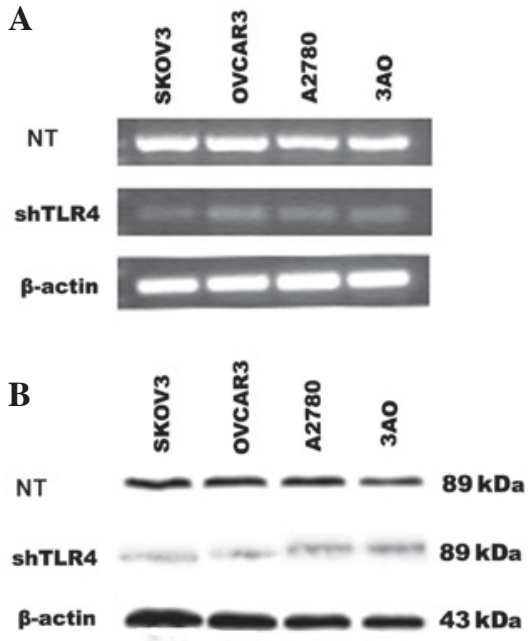

Figure 4. Effect of TLR4 knockdown in EOC cells using RT-PCR and western blot analysis. (A) Evaluation of mRNA expression of TLR4 in SKOV3, OVCAR3, A2780 and 3AO cell lines. (B) Western blot analysis for TLR4 protein expression in the four cell lines. shTLR4 cells were not treated with TLR4 interference vector transfection. TLR4, Toll-like receptor 4; EOC, epithelial ovarian cancer; RT-PCR, reverse transcription-polymerase chain reaction; NT, cells not treated with lipopolysaccharide or paclitaxel.

matory cytokines and growth factors. SKOV3/shTLR4 and OVCAR3/shTLR4 cells were found to secrete low levels of cytokines IL-6 and IL-8. However, these changes were not found in A2780/shTLR4 and 3AO/shTLR4. These results suggested that LPS/Pac results in a significant downregulation of IL-6 and IL-8 in MyD88+, but not in MyD88- cells, in which TLR4 had been knocked down (Figs. 5 and 6).

Effect of TLR4 on the Pac sensitivity of MyD $88^{+}$EOC cells. Of note, a positive increase in caspase-3/7 activity was identified between the MyD88 ${ }^{+}$and MyD88- cell lines (Fig. 7). In order to identify whether the MyD88 ${ }^{+}$cells respond to cell apoptosis through TLR4-MyD88 signaling, RNA interference was used to knock down the expression of TLR4 and the results are shown in Fig. 7. A significant increase was identified in caspase-3/7 activity following Pac treatment in SKOV3/shTLR4 cells compared with SKOV3/shControl cells $(\mathrm{P}<0.001)$, as was the case with OVCAR3 cells $(\mathrm{P}=0.000)$. No significant difference was observed in changes of caspase-3/7 activity between A2780/shTLR4 and A2780/shControl cells, as well as with $3 \mathrm{AO}$ cells. The results suggested that the TLR4-MyD88 signaling negatively regulates ovarian cancer cell sensitivity to Pac.

Knockdown of TLR4 depressed cell proliferation in MyD88 EOC cells. The effect of Pac on cell proliferation in SKOV3, OVCAR3, A2780 and 3AO cell lines was investigated. In Fig. 8, the growth inhibiting rate (GIR) of SKOV3/shTLR4 cells was $\sim 60 \%$ following $24 \mathrm{~h}$ treatment of $2 \mu \mathrm{M} \mathrm{Pac}$, which is significantly higher than those of the parental SKOV3 (29\%) and SKOV3/shControl $(31 \%)$ cells $(\mathrm{P}<0.001)$. In the OVCAR3 cell line, the same treatments were used as with the parental OVCAR3, OVCAR3/shControl and OVCAR3/shTLR4. The GIR was $\sim 61 \%$ in OVCAR3/shTLR4, which was higher than that of the parental OVCAR3 (33\%) and OVCAR3/shControl $(35 \%)(\mathrm{P}<0.001)$. However, no difference was observed in the 


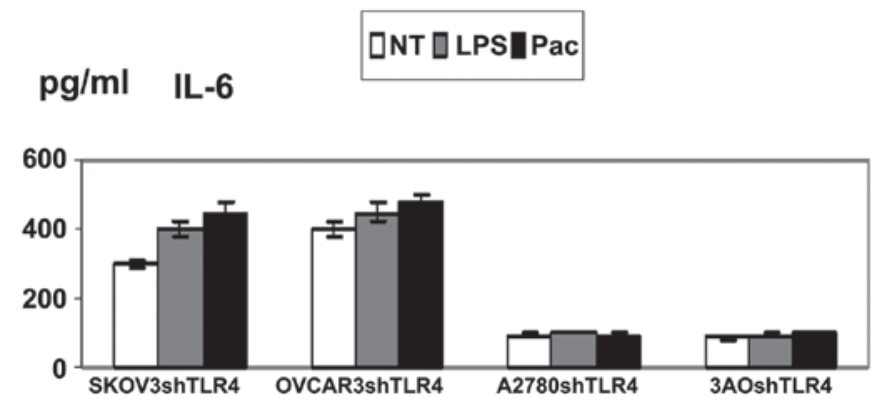

Figure 5. Levels of cytokines in tumor cell SNs. SNs were collected from tumor cells cultured at a density of $5 \times 10^{5}$ cells/well following $36 \mathrm{~h}$ of treatment and tested for levels of IL-6. SNs, supernatants; IL, interleukin; LPS, lipopolysaccharide; Pac, paclitaxel. NT, cells not treated with LPS or Pac.

\section{$\mathrm{pg} / \mathrm{ml}$ IL-8 $\quad$ QNT ULPSחPac}

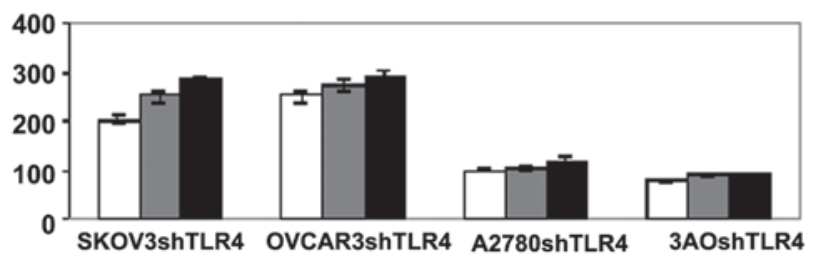

Figure 6. Levels of cytokines in tumor cell SNs. SNs were collected from tumor cells cultured at a density of $5 \times 10^{5}$ cells/well following $36 \mathrm{~h}$ of treatment and tested for levels of IL-8. SNs, supernatants; IL, interleukin; LPS, lipopolysaccharide; Pac, paclitaxel. NT, cells not treated with LPS or Pac.

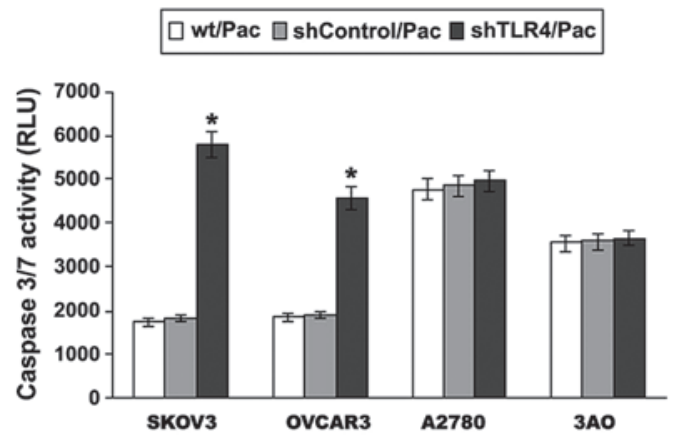

Figure 7. Following TLR4 knockdown, the apoptotic response of Pac $(2 \mu \mathrm{M})$ treatment in $\mathrm{MyD}^{+} 8^{+}$and MyD88- EOC cell lines was investigated. EOC cell lines were treated with $2 \mu \mathrm{mol} / 1$ Pac for $24 \mathrm{~h}$ and the levels of caspase-3/7 were measured using the Caspase-Glo 3/7 assay. Data are presented as the mean \pm SD from at least three independent experiments. A significant increase was identified in caspase-3/7 activity following Pac treatment in SKOV3/shTLR4 cells compared with SKOV3 and SKOV3/shControl cells, as was the case with the OVCAR3 cells. "P<0.001, vs. wt/Pac and shControl/Pac. TLR4, Toll-like receptor 4; MyD88, myeloid differentiation factor 88; EOC, epithelial ovarian cancer; Pac, paclitaxel; wt/Pac, SKOV3/OVCAR3/A2780/3AO parental cells treated with Pac; shControl/Pac, SKOV3/OVCAR3/A2780/3AO shControl cells treated with Pac; shTLR4/Pac, SKOV3/OVCAR3/A2780/3AO shTLR4 cells treated with Pac.

proliferation among the three types of A2780 cells (parental A2780, A2780/shControl and A2780/shTLR4), as well as in the 3AO cells (parental A2780, A2780/shControl and A2780/shTLR4). These results demonstrated that knockdown of TLR4 significantly restores the sensitivity of Pac in MyD88 ${ }^{+}$ ovarian cancer cells.

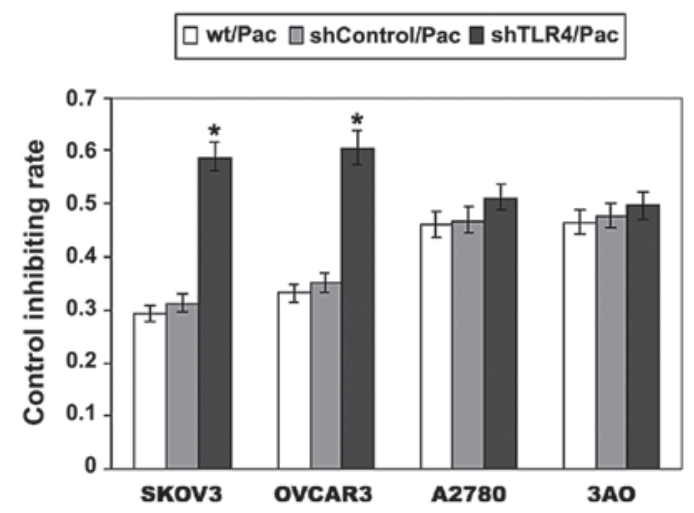

Figure 8. Effect of TLR4 knockdown on EOC cell growth using MTT assay. SKOV3/shTLR4 cells were inhibited by $\sim 60 \%$ in the presence of Pac at $2 \mu \mathrm{mol} / 1$ compared with parental SKOV3 (29\%) and SKOV3/shControl (31\%) cells. OVCAR3/shTLR4 cells showed a marked change compared with parental OVCAR3 and OVCAR3/shControl cells. However, no significant changes were identified among the parental, shControl and shTLR4 cells in the $\mathrm{A} 2780$ and $3 \mathrm{AO}$ cell lines. ${ }^{*} \mathrm{P}<0.001$, vs. wt/Pac and shControl/Pac. TLR4, Toll-like receptor 4; EOC, epithelial ovarian cancer; Pac, paclitaxel; wt/Pac, SKOV3/OVCAR3/A2780/3AO parental cells treated with Pac; shControl/Pac, SKOV3/OVCAR3/A2780/3AO shControl cells treated with Pac; shTLR4/Pac, SKOV3/OVCAR3/A2780/3AO shTLR4 cells treated with Pac.

\section{Discussion}

The successful treatment of ovarian cancer remains a major challenge. Overall, $>85 \%$ patients presenting with advanced disease are likely to relapse. Recurrence defines incurable disease in the majority of cases. The main obstacle to effective treatment is the failure of initial therapy to eradicate a sufficient number of tumor cells to prevent disease recurrence. Pac is a product of the Pacific yew and its antimitotic actions are due to its ability to bind and stabilize microtubules, which prevent accurate cell division during mitosis $(22,23)$. Pac induces the secretion of inflammatory cytokines in murine macrophages in a TLR4-dependent manner in addition to its antitumor effects. Although, the effect of Pac on human macrophages is controversial (24). Few previous studies have analyzed the contribution of innate immunity pathways to the mechanism of action of Pac (25). Pac is a first-line chemotherapeutic agent used in the treatment of EOC as well as recurrent EOC (26), and is known to be a TLR4 ligand $(27,28)$. In the present study, it was identified that Pac activates TLR4 signaling, which increases ovarian cancer cell proliferation.

Previously, MyD88 has been reported to be a negative regulator of TLR signaling $(29,30,31)$. It has been identified that MyD88 is an essential downstream component of the TLR4 signaling cascade mediating Pac resistance (19). In addition, it has been reported that LPS-stimulated tumor cell supernatants inhibit $\mathrm{T}$ cell proliferation and natural killer cell activity. Blockade of the TLR4 pathway reverses the functions of these cells in vitro and in vivo, delays tumor growth and, thus, prolongs the survival of tumor-bearing mice (32). In the present study, TLR4 was found to exhibit moderate $(++)$ or strong $(+++)$ expression in malignant $(20 / 24)$ and borderline (5/8) tumors and normal ovarian epithelium (6/12). The expression of MyD88 in malignant tumors was consid- 
erably greater $(18 / 24)$ than that in normal ovarian tissue $(1 / 8)$ or borderline tumors (1/12). The results of the present study support that MyD88 acts as a downstream factor and combines with TLR4 to increase the proliferation of ovarian cancer cells.

If TLR4 signaling highlights a survival benefit to tumor cells and alters their sensitivity to Pac, then its silencing via siRNA must aid in identifying the molecular mechanisms responsible for LPS- and Pac-mediated effects. We hypothesized that in TLR4-MyD88 signaling, TLR4 is activated by Pac. MyD88 $8^{+}$human ovarian carcinoma cells (SKOV3 and OVCAR3) and MyD88- ovarian carcinoma cell lines (A2780 and 3AO) were selected to investigate the TLR4 effects on apoptosis with Pac chemotherapy. The molecular mechanisms of chemotherapy resistance are considered to be associated with apoptosis inhibition $(33,34)$. Acquired resistance to chemotherapy is a significant impediment to effective cancer therapy (35). Notably, silencing of TLR4 expression in MyD88 ${ }^{+}$ EOC cells results in sensitization of the cells to Pac-induced apoptosis and this sensitization is accompanied by the inhibition of cytokine IL- 6 and IL- 8 production in response to Pac and LPS. In the current study, MyD88 ${ }^{+}$cells (SKOV3 and OVCAR3) constitutively secreted a wide range of cytokines including, IL-6 and IL-8. By contrast, MyD88 cells (A2780 and 3AO) produced low levels of these cytokines. LPS and Pac significantly increased the secretion of IL- 6 and IL-8 in SKOV3 and OVCAR3 cells, but not in A2780 and 3AO cells (Figs. 2 and 3).

The present study used RNA interference to knock down the expression of TLR4 in SKOV3, OVCAR3, A2780 and 3AO cell lines. Cytokine production in response to LPS and Pac stimulation was significantly inhibited in SKOV3/shTLR4 and OVCAR3/shTLR4 cells. No changes in cytokine production were observed in A2780/shTLR4 and 3AO/shTLR4 cells (Figs. 5 and 6).

Caspase-Glo 3/7 assay was used to investigate the apoptosis of ovarian cancer cells. When TLR4 was knocked down, a positive increase in caspase-3/7 activity was identified following Pac treatment between MyD88 ${ }^{+}$and MyD88 cell lines. The mechanism responsible for Pac resistance in ovarian cancer is not completely understood. The present study confirmed that there is a negative correlation between MyD88 expression and Pac-induced apoptosis in TLR4 signaling, consistent with the results of a previous study (36).

In addition, RNA interference was used to knockdown the expression of TLR4 in SKOV3, OVCAR3, A2780 and 3AO cell lines. In the caspase-Glo 3/7 assay, a significant increase of caspase-3/7 activity was identified in SKOV3/shTLR4 and in OVCAR3/shTLR4 cells (Fig. 7).

The results of the current study indicated that TLR4-MyD88 signaling negatively regulates Pac treatment. Knockdown of TLR4 may increase Pac chemosensitivity in MyD88 $8^{+}$cells. In addition to the caspase-Gol 3/7 assay, cell proliferation was evaluated and a significant increase of GIR was identified in SKOV3/shTLR4 and OVCAR3/shTLR4 cell lines. However, in A2780/shTLR4 and 3AO/shTLR4 cells, no significant changes were observed compared with their control cells (Fig. 8). The present study revealed that the proliferation and survival of the cancer cells is regulated by a specific defense mechanism in TLR4/MyD88 signaling.
In conclusion, the observations of the current study imply that Pac activates TLR4-MyD88 signaling, which increases ovarian cancer cell proliferation. Although the results suggest that the knockdown of TLR4 inhibits cell growth and that IL-6 and IL-8 levels are associated with MyD88 (+) EOC cells, the precise underlying molecular mechanisms responsible for these observations remain to be established. As TLR4 is functionally associated with tumor progression, TLR4 is likely to be a promising target for tumor therapy in the future.

\section{Acknowledgements}

The authors would like to thank Professor Pei-Shu Liu (Department of Obstetrics and Gynecology, Qilu Hospital, Shandong University, Jinan, China) for support.

\section{References}

1. Akira S, Uematsu S and Takeuchi O: Pathogen recognition and innate immunity. Cell 124: 783-801, 2006.

2. Takeda K, Kaisho T and Akira S: Toll-like receptors. Annu Rev Immunol 21: 335-376, 2003.

3. Yu L and Chen S: Toll-like receptors expressed in tumor cells: targets for therapy. Cancer Immunol Immunother 57: 1271-1278, 2008.

4. Medzhitov R, Preston-Hurlburt P and Janeway CA Jr: A human homologue of the Drosophila Toll protein signals activation of adaptive immunity. Nature 388: 394-397, 1997.

5. Poltorak A, He X, Smirnova I, et al: Defective LPS signaling in $\mathrm{C} 3 \mathrm{H} / \mathrm{HeJ}$ and $\mathrm{C} 57 \mathrm{BL} / 10 \mathrm{ScCr}$ mice: mutations in Tlr4 gene. Science 282: 2085-2088, 1998.

6. Pathak SK, Basu S, Bhattacharyya A, Pathak S, Kundu M and Basu J: Mycobacterium tuberculosis lipoarabinomannan-mediated IRAK-M induction negatively regulates Toll-like receptor-dependent interleukin- 12 p40 production in macrophages. J Biol Chem 280: 42794-42800, 2005.

7. He W, Liu Q, Wang L, Chen W, Li N and Cao X: TLR4 signaling promotes immune escape of human lung cancer cells by inducing immunosuppressive cytokines and apoptosis resistance. Mol Immunol 44: 2850-2859, 2007.

8. Fallows S, Price J, Atkinson RJ, Johnston PG, Hickey I and Russell SE: P53 mutation does not affect prognosis in ovarian epithelial malignancies. J Pathol 194: 68-75, 2001.

9. Byrd-Leifer CA, Block EF, Takeda K, Akira S and Ding A: The role of MyD88 and TLR4 in the LPS-mimetic activity of Taxol. Eur J Immunol 31: 2448-2457, 2001.

10. Lord KA, Hoffman-Liebermann B and Liebermann DA: Nucleotide sequence and expression of a cDNA encoding MyD88, a novel myeloid differentiation primary response gene induced by IL6. Oncogene 5: 1095-1097, 1990.

11. Asschert JG, Vellenga E, Ruiters MH and de Vries EG: Regulation of spontaneous and TNF/IFN-induced IL- 6 expression in two human ovarian-carcinoma cell lines. Int J Cancer 82: 244-249, 1999.

12. Watson JM, Sensintaffar JL, Berek JS and Martinez-Maza O: Constitutive production of interleukin 6 by ovarian cancer cell lines and by primary ovarian tumor cultures. Cancer Res 50: 6959-6965, 1990.

13. Wu S, Rodabaugh K, Martinez-Maza O, et al: Stimulation of ovarian tumor cell proliferation with monocyte products inducing interleukin 1, interleukin 6 and tumor necrosis factor-alpha. Am J Obstet Gynecol 166: 977-1007, 1992.

14. Obata NH, Tamakoshi K, Shibata K, Kikkawa F and Tomoda Y: Effects of interleukin-6 on in vitro cell attachment, migration and invasion of human ovarian carcinoma. Anticancer Res 17: 337-342, 1997.

15. Nilsson MB, Langley RR and Fidler IJ: Interleukin-6, secreted by human ovarian carcinoma cells, is a potent proangiogenic cytokine. Cancer Res 65: 10794-10800, 2005.

16. Shahzad MM, Arevalo JM, Armaiz-Pena GN, et al: Stress effects on FosB- and interleukin-8 (IL8)-driven ovarian cancer growth and metastasis. J Biol Chem 285: 35462-35470, 2010. 
17. Kawai T, Adachi O, Ogawa T, Takeda $\mathrm{K}$ and Akira S: Unresponsiveness of MyD88-deficient mice to endotoxin. Immunity 11: 115-122, 1999.

18. Björkbacka H, Fitzgerald KA, Huet F, et al: The induction of macrophage gene expression by LPS predominantly utilizes Myd88-independent signaling cascades. Physiol Genomics 19: 319-330, 2004.

19. Kelly MG, Alvero AB, Chen R, et al: TLR-4 signaling promotes tumor growth and Pac chemoresistance in ovarian cancer. Cancer Res 66: 3859-3868, 2006.

20. Wang AC, Su QB, Wu FX, Zhang XL and Liu PS: Role of TLR4 for Pac chemotherapy in human epithelial ovarian cancer cells. Eur J Clin Invest 39: 157-164, 2009.

21. Mao HL, Liu PS, Zheng JF, et al: Transfection of Smac/DIABLO sensitizes drug-resistant tumor cells to TRAIL or Pac-induced apoptosis in vitro. Pharmacol Res 56: 483-492, 2007.

22. Wani MC, Taylor HL, Wall ME, Coggon P and McPhail AT: Plant antitumor agents. VI. The isolation and structure of taxol, a novel antileukemic and antitumor agent from Taxus brevifolia. J Am Chem Soc 93: 2325-2327, 1971.

23. Manfredi JJ, Parness J and Horwitz SB: Taxol binds to cellular microtubules. J Cell Biol 94: 688-696, 1982.

24. Wang J, Kobayashi M, Han M, et al: MyD88 is involved in thesignalling pathway for Taxol-induced apoptosis and TNF-alpha expression in human myelomonocytic cells. Br J Haematol 118 638-645, 2002.

25. Zimmer SM, Liu J, Clayton JL, Stephens DS and Snyder JP: Paclitaxelbinding to human and murine MD-2. J Biol Chem 283 27916-27926, 2008

26. Ozols RF, Bundy BN, Greer BE, et al: Phase III trial of carboplatin and Paclitaxel compared with cisplatin and Pac in patients with optimally resected stage III ovarian cancer: a Gynecologic Oncology Group study. J Clin Oncol 21: 3194-3200, 2003.
27. Song $\mathrm{G}$, Ouyang $\mathrm{G}$ and Bao $\mathrm{S}$ : The activation of Akt/PKB signaling pathway and cell survival. J Cell Mol Med 9: 59-71, 2005.

28. Ding AH, Porteu F, Sanchez E and Nathan CF: Shared actions of endotoxin and taxol on TNF receptors and TNF release. Science 248: 370-372, 1990.

29. Brint EK, Xu D, Liu H, et al: ST2 is an inhibitor of interleukin 1 receptor and Toll-like receptor 4 signaling and maintains endotoxin tolerance. Nat Immunol 5: 373-379, 2004.

30. Burns K, Janssens S, Brissoni B, Olivos N, Beyaert R and Tschopp J: Inhibition of interleukin 1 receptor/Toll-like receptor signaling through the alternatively spliced, short form of MyD88 is due to its failure to recruit IRAK-4. J Exp Med 197: 263-268, 2003.

31. Schmitz I, Kirchhoff S and Krammer PH: Regulation of death receptor-mediated apoptosis pathways. Int J Biochem Cell Biol 32: 1123-1136, 2000.

32. Huang B, Zhao J, Li H, et al: Toll-like receptors on tumor cells facilitate evasion of immune surveillance. Cancer Res 65: 5009-5014, 2005.

33. Pérez-Tomás R: Multidrug resistance: retrospect and prospects in anti-cancer drug treatment. Curr Med Chem 13: 1859-1876, 2006.

34. Fulda S and Debatin KM: Extrinsic versus intrinsic apoptosis pathways in anticancer chemotherapy. Oncogene 25: 4798-4811, 2006.

35. Nakanishi $\mathrm{C}$ and Toi M: Nuclear factor-kappaB inhibitors as sensitizers to anticancer drugs. Nat Rev Cancer 5: 297-309, 2005.

36. Silasi DA, Alvero AB, Illuzzi J, et al: MyD88 predicts chemoresistance to Pac in epithelial ovarian cancer. Yale J Biol Med 79: $153-163,2006$. 